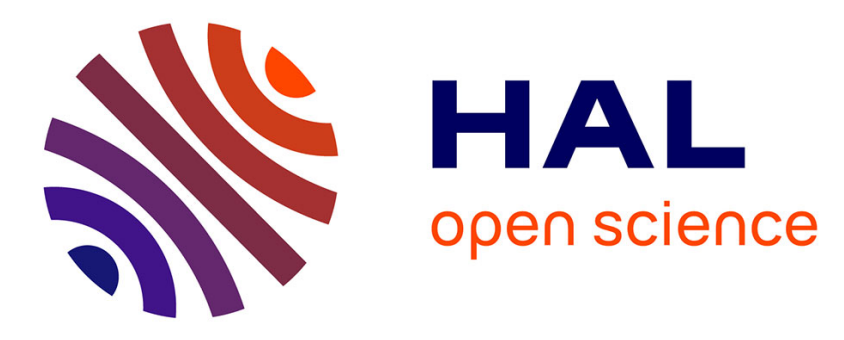

\title{
Satisfiability Modulo Theories and Assignments
}

Maria Paola Bonacina, Stéphane Graham-Lengrand, Natarajan Shankar

\section{To cite this version:}

Maria Paola Bonacina, Stéphane Graham-Lengrand, Natarajan Shankar. Satisfiability Modulo Theories and Assignments. CADE 2017 - 26th International Conference on Automated Deduction, Aug 2017, Gothenburg, Sweden. 10.1007/978-3-319-63046-5_4 . hal-01615830

\section{HAL Id: hal-01615830 \\ https://hal.science/hal-01615830}

Submitted on 12 Oct 2017

HAL is a multi-disciplinary open access archive for the deposit and dissemination of scientific research documents, whether they are published or not. The documents may come from teaching and research institutions in France or abroad, or from public or private research centers.
L'archive ouverte pluridisciplinaire HAL, est destinée au dépôt et à la diffusion de documents scientifiques de niveau recherche, publiés ou non, émanant des établissements d'enseignement et de recherche français ou étrangers, des laboratoires publics ou privés. 


\title{
Satisfiability Modulo Theories and Assignments
}

\author{
Maria Paola Bonacina ${ }^{1}$, Stéphane Graham-Lengrand ${ }^{2,3}$, and Natarajan \\ Shankar ${ }^{2}$ \\ 1 Università degli Studi di Verona, Verona, Italy \\ 2 SRI International, Menlo Park, USA \\ 3 CNRS - INRIA - École Polytechnique, Palaiseau, France
}

\begin{abstract}
The CDCL procedure for SAT is the archetype of conflictdriven procedures for satisfiability of quantifier-free problems in a single theory. In this paper we lift CDCL to CDSAT (Conflict-Driven Satisfiability), a system for conflict-driven reasoning in combinations of disjoint theories. CDSAT combines theory modules that interact through a global trail representing a candidate model by Boolean and first-order assignments. CDSAT generalizes to generic theory combinations the modelconstructing satisfiability calculus (MCSAT) introduced by de Moura and Jovanović. Furthermore, CDSAT generalizes the equality sharing (Nelson-Oppen) approach to theory combination, by allowing theories to share equality information both explicitly through equalities and disequalities, and implicitly through assignments. We identify sufficient conditions for the soundness, completeness, and termination of CDSAT.
\end{abstract}

Keywords: Theory combination - Conflict-driven decision procedures · Model building. Satisfiability modulo assignment

\section{Introduction}

A growing trend in automated deduction is the generalization of conflict-driven reasoning from propositional to first-order logic (cf. [2] for a brief coeval survey). For propositional satisfiability (SAT), the conflict-driven clause learning (CDCL) procedure works by guessing assignments to variables, propagating their consequences through clauses, and learning new clauses, or lemmas, when assignments lead to conflicts [14]. The conflict-driven paradigm has been extended to decide the $\mathcal{T}$-satisfiability of sets of literals when $\mathcal{T}$ is one of several fragments of arithmetic $[4,7,10,11,12,15,17,18]$. Key features of such conflict-driven $\mathcal{T}$-satisfiability procedures are the use of assignments to first-order variables and the explanation of conflicts with lemmas, which may contain atoms that are not in the input. We illustrate these features by an example. Consider the following set of literals, which is unsatisfiable in Linear Rational Arithmetic (LRA):

$$
R=\left\{l_{0}:(-2 \cdot x-y<0), \quad l_{1}:(x+y<0), \quad l_{2}:(x<-1)\right\} .
$$

A conflict-driven LRA-satisfiability procedure attempts to build a model by guessing a value for one of the variables, say $y \leftarrow 0$. This lets $l_{0}$ yield the lower 
bound $x>0$. Given the upper bound $l_{2}$, the space of possible values for $x$ is empty, revealing that the guess and the constraints are in conflict. The procedure explains the conflict by the new atom $l_{3}:(-y<-2)$, the linear combination of $l_{0}$ and $l_{2}$ that eliminates $x$. This excludes not only $y \leftarrow 0$, but also all assignments $y \leftarrow \mathfrak{c}$ where $\mathfrak{c} \leq 2$. Suppose the procedure retracts the assignment $y \leftarrow 0$ and tries $y \leftarrow 4$. This lets $l_{1}$ yield the upper bound $x<-4$, and $l_{0}$ the lower bound $x>-2$. The procedure explains this conflict by the new atom $l_{4}:(y<0)$, the linear combination of $l_{0}$ and $l_{1}$ that eliminates $x$. As $l_{4}$ is violated by the assignment $y \leftarrow 4$, the procedure retracts $y \leftarrow 4$. Then no assignment to $y$ can satisfy both $l_{3}$ and $l_{4}$. This third conflict is explained by the linear combination of $l_{3}$ and $l_{4}$ that eliminates $y$, namely $0<-2$, which is also a new atom. Since this consequence of the original problem is a contradiction, the procedure returns unsatisfiable.

Applications typically require deciding the satisfiability of arbitrary quantifier-free formulae, or, equivalently, sets of ground clauses, in a combination $\mathcal{T}$ of theories $\mathcal{T}_{1}, \ldots, \mathcal{T}_{n}$. The $\operatorname{DPLL}(\mathcal{T})$ approach $[1,13]$ combines a CDCL-based SAT-solver with a $\mathcal{T}$-satisfiability procedure, obtained from $\mathcal{T}_{i}$-satisfiability procedures by the equality sharing method [16], assuming that the theories $\mathcal{T}_{i}$ are disjoint and stably infinite: they do not share symbols other than equality and admit countably infinite models. The $\mathcal{T}_{i}$-satisfiability procedures are combined as black-boxes that only propagate equalities between shared variables. $\operatorname{DPLL}(\mathcal{T})$ uses their combination as a black-box that only detects $\mathcal{T}$-conflicts and propagates $\mathcal{T}$-lemmas, while the SAT-solver tries assignments and builds a candidate model. If conflict-driven $\mathcal{T}_{i}$-satisfiability procedures were integrated in this manner, the combination would not be conflict-driven. To make it conflict-driven, the $\mathcal{T}_{i}$-satisfiability procedures need to cooperate to build a model, sharing assignments and exporting lemmas to explain conflicts, possibly using new atoms.

MCSAT, for Model-Constructing Satisfiability, is a paradigm for integrating a CDCL-based SAT-solver with a conflict-driven $\mathcal{T}$-satisfiability procedure [5]. MCSAT uses first-order assignments on a par with Boolean ones, coordinates the conflict explanation mechanisms at the Boolean and theory levels in a unified manner, and incorporates the capability of creating new atoms. MCSAT lifted CDCL to SMT in the sense of satisfiability modulo a single theory $\mathcal{T}$, which was instantiated to the theory of bit-vectors [19] and to non-linear integer arithmetic [8]. A version of MCSAT was also given for the specific combination of LRA and Equality with Uninterpreted Function symbols (EUF) [9], but the conflict-driven combination of a generic range of theories remained an open problem.

In this paper we generalize conflict-driven reasoning to generic combinations of disjoint theories, solving the problem of combining multiple conflict-driven $\mathcal{T}_{i}$-satisfiability procedures into a conflict-driven $\mathcal{T}$-satisfiability procedure. We introduce a new method for theory combination, called CDSAT for ConflictDriven Satisfiability. For example, it decides the satisfiability of problems in the combination of LRA, EUF and the theory of arrays, such as

$$
P=\{f(\operatorname{select}(\operatorname{store}(a, i, v), j)) \simeq w, f(u) \simeq w-2, i \simeq j, u \simeq v\} .
$$

CDSAT treats propositional and theory reasoning uniformly: formulae are terms of sort prop (for proposition); propositional logic is one of the theories $\mathcal{T}_{1}, \ldots, \mathcal{T}_{n}$; 
and CDCL is one of the $\mathcal{T}_{i}$-satisfiability procedures to be combined. With formulae reduced to terms, assignments become the data manipulated by inferences. CDSAT combines $\mathcal{T}_{i}$-inference systems, called theory modules [6], rather than $\mathcal{T}_{i^{-}}$ satisfiability procedures. Ideally, a $\mathcal{T}_{i}$-satisfiability procedure, like any reasoning procedure, is defined by an inference system and a search plan. Since all conflictdriven procedures have the same conflict-driven search plan, what needs to be combined are the inference systems, while the common conflict-driven control is factored out and handled centrally by CDSAT. We prove that CDSAT is sound, complete, and terminating, identifying the sufficient conditions that theories and theory modules need to fulfill for these properties to hold.

We believe that the abstraction of viewing combination of theories as combination of inference systems, rather than procedures, allows us to bring simplicity and elegance to theory combination. A $\mathcal{T}_{i}$-satisfiability procedure that is not conflict-driven can still be integrated in CDSAT by treating it as a theory module whose only inference rule invokes the $\mathcal{T}_{i}$-satisfiability procedure to detect the $\mathcal{T}_{i}$-unsatisfiability of a set of assignments. Therefore CDSAT subsumes both MCSAT and equality sharing. CDSAT reduces to MCSAT, if there are only propositional logic with CDCL and another theory $\mathcal{T}$ with a conflict-driven $\mathcal{T}$-satisfiability procedure. CDSAT reduces to equality sharing, if none of the theories has a conflict-driven $\mathcal{T}$-satisfiability procedure.

\section{Preliminaries}

We assume the basic definitions of multi-sorted first-order logic. A signature $\Sigma=(S, F)$ consists of a set $S$ of sorts, including prop, and a set $F$ of symbols, including a collection $\simeq_{S}$ of equality symbols $\simeq_{s}:(s \times s) \rightarrow$ prop for every $s \in S$. Sorts can be omitted when clear from context. The other symbols in $F$ may be constant, function, and predicate symbols, as well as logical connectives such as $\wedge, \vee$, and $\neg$. Given a class $\mathcal{V}=\left(\mathcal{V}^{s}\right)_{s \in S}$ of sets of sorted variables, $\Sigma[\mathcal{V}]$-terms are defined as usual, and formulae are terms of sort prop. We use $l$ for formulae and $t$ and $u$ for terms of any sort. Formulae in the standard sense are obtained as the closure of our formulae under quantifiers and logical connectives; $\Sigma$-sentences are those with no free variables.

A $\Sigma[\mathcal{V}]$-interpretation $\mathcal{M}$ interprets each sort $s$ in $S$ as a non-empty set $s^{\mathcal{M}}$ with prop ${ }^{\mathcal{M}}=\left\{\right.$ true, false ; each symbol $f:\left(s_{1} \times \cdots \times s_{m}\right) \rightarrow s$ in $F$ as a function $f^{\mathcal{M}}: s_{1}^{\mathcal{M}} \times \cdots \times s_{m}^{\mathcal{M}} \rightarrow s^{\mathcal{M}}$ with $\simeq_{s}^{\mathcal{M}}$ returning true if and only if its arguments are identical; and each variable $v \in \mathcal{V}^{s}$ as an element $v^{\mathcal{M}} \in s^{\mathcal{M}}$. The interpretation $\mathcal{M}(t)$ of a $\Sigma[\mathcal{V}]$-term $t$ is defined as usual. $\Sigma[\emptyset]$-interpretations, known as $\Sigma$-structures, suffice for $\Sigma$-sentences.

A theory $\mathcal{T}$ on signature $\Sigma$ is defined axiomatically as a set of $\Sigma$-sentences, called its axioms, or model-theoretically as the class of $\Sigma$-structures, called $\mathcal{T}$ models, that satisfy the axioms of $\mathcal{T}$. A $\mathcal{T}[\mathcal{V}]$-model is any $\Sigma[\mathcal{V}]$-interpretation whose underlying $\Sigma$-structure is a $\mathcal{T}$-model.

Let $\mathcal{T}_{1}, \ldots, \mathcal{T}_{n}$ be disjoint theories with signatures $\Sigma_{1}=\left(S_{1}, F_{1}\right), \ldots$, $\Sigma_{n}=\left(S_{n}, F_{n}\right)$ : they do not share symbols other than equality, but can share 
sorts, meaning that $F_{i} \cap F_{j}=\left(\simeq_{S_{i} \cap S_{j}}\right)$ for $i \neq j$. Let $\mathcal{T}_{\infty}$ be their union, with signature $\Sigma_{\infty}=\left(S_{\infty}, F_{\infty}\right)$ for $S_{\infty}=\bigcup_{k=1}^{n} S_{k}$ and $F_{\infty}=\bigcup_{k=1}^{n} F_{k}$, and axiomatization given by the union of those of $\mathcal{T}_{1}, \ldots, \mathcal{T}_{n}$. We fix a global collection of variables $\mathcal{V}_{\infty}=\left(\mathcal{V}_{\infty}^{s}\right)_{s \in S_{\infty}}$, use variables for variables in $\mathcal{V}_{\infty}$, and terms for $\Sigma_{\infty}\left[\mathcal{V}_{\infty}\right]$-terms.

Example 1. Problem $P$ from Sect. 1 is written in the signatures

$$
\begin{aligned}
& \Sigma_{\mathrm{LRA}}=\left(\{\text { prop, } \mathrm{Q}\}, \simeq_{\{\text {prop }, \mathrm{Q}\}} \cup\{0,1: \mathrm{Q},+: \mathrm{Q} \times \mathrm{Q} \rightarrow \mathrm{Q}\} \cup\{q \cdot: \mathrm{Q} \rightarrow \mathrm{Q} \mid q \in \mathbb{Q}\}\right) \\
& \Sigma_{\text {EUF }}=\left(\{\text { prop, Q }, V\}, \simeq_{\{\text {prop }, Q, V\}} \cup\{f: V \rightarrow \mathrm{Q}\}\right) \\
& \left.\Sigma_{\text {Arr }}=(\text { prop, } V, I, A\}, \simeq_{\{\text {prop }, V, I, A\}} \cup\{\text { select }: A \times I \rightarrow V \text {, store }: A \times I \times V \rightarrow A\}\right)
\end{aligned}
$$

where $Q$ and $\mathbb{Q}$ are the sort and the set of the rationals, $q \cdot$ is scalar multiplication, and $A, I$, and $V$ are the sorts of arrays, indices, and values.

\section{Assignments and Theory Modules}

CDSAT solves $\mathcal{T}_{\infty}$-satisfiability problems presented as assignments of values to terms. For example, a set of formulae $\left\{l_{1}, \ldots, l_{m}\right\}$ is represented as the assignment $\left\{l_{1} \leftarrow\right.$ true $, \ldots, l_{m} \leftarrow$ true $\}$. The input assignment may contain terms of any sort. Assignments are public, meaning visible to all $\mathcal{T}_{k}$-inference systems.

\subsection{Assignments}

We need to identify the language of values that the system can assign to terms, besides true and false. Assignable values are not necessarily in the theories' signatures (e.g., consider $x \leftarrow \sqrt{2}$ for the sort of the reals). Therefore, we introduce for each theory $\mathcal{T}_{k}, 1 \leq k \leq n$, a conservative extension $\mathcal{T}_{k}^{+}$with signature $\Sigma_{k}^{+}=\left(S_{k}, F_{k}^{+}\right)$, where $F_{k}^{+}$is the extension of $F_{k}$ with a possibly empty set of new constant symbols called $\mathcal{T}_{k}^{+}$-values. An extension is conservative if any $\mathcal{T}_{k}^{+}$-unsatisfiable set of $\Sigma_{k}[\mathcal{V}]$-formulae is also $\mathcal{T}_{k}$-unsatisfiable. This ensures that reasoning in the extension does not change the problem: if CDSAT discovers $\mathcal{T}_{k}^{+}$unsatisfiability, the problem is $\mathcal{T}_{k}$-unsatisfiable; if the problem is $\mathcal{T}_{k}$-satisfiable, there is a $\mathcal{T}_{k}^{+}$-model that CDSAT can build. A sort $s \in S_{k}$ with at least one $\mathcal{T}_{k}^{+}$-value is called $\mathcal{T}_{k}$-public, as a term of sort $s$ may be assigned a $\mathcal{T}_{k}^{+}$-value. A sort $s$ may be $\mathcal{T}_{i}$-public and $\mathcal{T}_{j}$-public for $i \neq j$. We stipulate that sort prop is $\mathcal{T}_{k^{-}}$ public for all $k$, with $\mathcal{T}_{k}^{+}$-values true and false, which are valid and unsatisfiable, respectively, in $\mathcal{T}_{k}^{+}$. We use $\mathfrak{b}$ for true or false. We assume that the extended theories are still disjoint except for the two Boolean values true and false.

Example 2. Let $\mathrm{RA}$ be the theory of real arithmetic with sorts $\{\mathrm{R}$, prop $\}$ and symbols $\simeq_{\{\mathrm{R}, \text { prop }\}} \cup\{0,1: \mathrm{R} ;+,-, \cdot: \mathrm{R} \times \mathrm{R} \rightarrow \mathrm{R}\} . \mathrm{RA}^{+}$adds a new constant for every real number, so $R$ is RA-public. The axioms of $R^{+}$are the formulae that hold in the standard model of the reals interpreting every $\mathrm{RA}^{+}$-value as itself.

Extending the signature with names that denote all individuals in the domain of a $\mathcal{T}_{k}$-model is a standard move in automated reasoning, where models need to be built out of syntax, and especially when $\mathcal{T}_{k}$ has an "intended model" as in arithmetic. In such cases a $\mathcal{T}_{k}^{+}$-value is both the domain element and the constant symbol that names it. 


$$
\begin{aligned}
& t_{1} \leftarrow \mathfrak{c}_{1}, t_{2} \leftarrow \mathfrak{c}_{2} \vdash t_{1} \simeq_{s} t_{2} \text { if } \mathfrak{c}_{1} \text { and } \mathfrak{c}_{2} \text { are the same } \mathcal{T}^{+} \text {-value of sort } s \\
& t_{1} \leftarrow \mathfrak{c}_{1}, t_{2} \leftarrow \mathfrak{c}_{2} \vdash t_{1} \varkappa_{s} t_{2} \text { if } \mathfrak{c}_{1} \text { and } \mathfrak{c}_{2} \text { are distinct } \mathcal{T}^{+} \text {-values of sort } s \\
& \vdash t_{1} \simeq_{s} t_{1} \\
& t_{1} \simeq_{s} t_{2} \vdash t_{2} \simeq_{s} t_{1} \\
& t_{1} \simeq_{s} t_{2}, t_{2} \simeq_{s} t_{3} \vdash t_{1} \simeq_{s} t_{3}
\end{aligned}
$$

Fig. 1. Equality inference rules: $t_{1}, t_{2}$, and $t_{3}$ are terms of sort $s$

Definition 1 (Assignment). Given a theory $\mathcal{T}$ with extension $\mathcal{T}^{+}$, a $\mathcal{T}$-assignment is a set of pairs $t \leftarrow \mathfrak{c}$, where $t$ is a term and $\mathfrak{c}$ is a $\mathcal{T}^{+}$-value of the same sort. Term $t$ and all its subterms are said to occur in the assignment. An assignment is plausible if it does not contain both $l \leftarrow$ true and $l \leftarrow$ false for any formula $l$.

For example, $\{x \leftarrow \sqrt{2}, x+y \leftarrow \sqrt{3}\}$ and $\{f(x) \leftarrow \sqrt{2},(1 \cdot x \simeq x) \leftarrow$ true $\}$ are RA-assignments. If for all pairs $t \leftarrow \mathfrak{c}$ the sort of $t$ and $\mathfrak{c}$ is $s$, the $\mathcal{T}$-assignment is of sort $s$, and if $s=$ prop it is Boolean. A first-order $\mathcal{T}$-assignment is a $\mathcal{T}$-assignment that is not Boolean. We use $J$ for generic $\mathcal{T}$-assignments, $A$ for singleton ones, and $L$ for Boolean singletons. We abbreviate $l \leftarrow$ true as $l, l \leftarrow$ false as $\bar{l}$, and $t \simeq_{s} u \leftarrow$ false as $t \chi_{s} u$. The flip $\bar{L}$ of $L$ assigns to the same formula the opposite Boolean value. The union of $\mathcal{T}_{1}^{+}, \ldots, \mathcal{T}_{n}^{+}$is an extension $\mathcal{T}_{\infty}^{+}$of $\mathcal{T}_{\infty}$, with signature $\Sigma_{\infty}^{+}=\left(S_{\infty}, F_{\infty}^{+}\right)$for $F_{\infty}^{+}=\bigcup_{k=1}^{n} F_{k}^{+}$. We use $H$ for $\mathcal{T}_{\infty}$ assignments, called assignments for short. Plausibility does not forbid an assignment $\{t \leftarrow 3.1, u \leftarrow 5.4, t \leftarrow$ red, $u \leftarrow$ blue $\}$, where the first two pairs are $\mathcal{T}_{1}$ assignments and the last two are $\mathcal{T}_{2}$-assignments; the sort of $t$ and $u$ is both $\mathcal{T}_{1}$-public and $\mathcal{T}_{2}$-public. When building a model from this assignment, 3.1 will be identified with red and 5.4 with blue.

Definition 2 (Theory view). Let $\mathcal{T}$ and $S$ be either $\mathcal{T}_{\infty}$ and $S_{\infty}$, or $\mathcal{T}_{k}$ and $S_{k}$ for $1 \leq k \leq n$. The $\mathcal{T}$-view of an assignment $H$ is the $\mathcal{T}$-assignment $H_{\mathcal{T}}=$

$$
\{t \leftarrow \mathfrak{c} \quad \mid t \leftarrow \mathfrak{c} \text { is a } \mathcal{T} \text {-assignment in } H\} \cup
$$

$\bigcup_{k=1}^{n}\left(\left\{t_{1} \simeq_{s} t_{2} \mid t_{1} \leftarrow \mathfrak{c}, t_{2} \leftarrow \mathfrak{c}\right.\right.$ are $\mathcal{T}_{k}$-assignments in $H$ of sort $s \in S \backslash\{$ prop $\left.\}\right\} \cup$

$\left\{t_{1} \Varangle_{s} t_{2} \mid t_{1} \leftarrow \mathfrak{c}_{1}, t_{2} \leftarrow \mathfrak{c}_{2}\right.$ are $\mathcal{T}_{k}$-assignments in $H$ of sort $s \in S \backslash\{$ prop $\left.\left.\}, \mathfrak{c}_{1} \neq \mathfrak{c}_{2}\right\}\right)$.

$H_{\mathcal{T}}$ contains the $\mathcal{T}$-assignments of $H$, plus all equalities and disequalities induced by the $\mathcal{T}_{k}$-assignments in $H, 1 \leq k \leq n$. We introduce next theory modules, the abstract counterpart of theory solvers or theory plugins [9].

\subsection{Theory Modules}

A theory module $\mathcal{I}$ for theory $\mathcal{T}$ is an inference system whose inferences, called $\mathcal{I}$-inferences and written $J \vdash_{\mathcal{I}} L$, derive a singleton Boolean assignment $L$ from a $\mathcal{T}$-assignment $J$. Since all theories include equality, all theory modules include the equality inference rules of Fig. 1 . The following inferences are $\mathcal{I}_{\mathrm{RA}}$-inferences:

$$
\begin{aligned}
& (x \leftarrow \sqrt{2}),(y \leftarrow \sqrt{2}) \vdash_{\mathcal{I}_{\mathrm{RA}}}(x \cdot y \simeq 1+1) \\
& (y \leftarrow \sqrt{2}),(x \leftarrow \sqrt{2}) \vdash_{\mathcal{I}_{\mathrm{RA}}}(y \simeq x) \\
& (y \leftarrow \sqrt{2}),(x \leftarrow \sqrt{3}) \vdash_{\mathcal{I}_{\mathrm{RA}}}(y \nsucceq x)
\end{aligned}
$$


$\mathcal{I}$-inferences only derive Boolean assignments because CDSAT does not justify first-order assignments by inferences, not even when a first-order assignment is forced by others (e.g., $y \leftarrow 2$ by $x \leftarrow 1$ and $(x+y) \leftarrow 3$ ). We assume we have theory modules $\mathcal{I}_{1}, \ldots, \mathcal{I}_{n}$ for $\mathcal{T}_{1}, \ldots, \mathcal{T}_{n}$. We now define acceptability and relevance.

Definition 3 (Acceptability). Given $\mathcal{T}_{k}$-assignments $t \leftarrow \mathfrak{c}$ and $J, t \leftarrow \mathfrak{c}$ is acceptable for $J$ and $\mathcal{I}_{k}$, if (i) $J$ does not assign a value to $t$ and (ii) either $t \leftarrow \mathfrak{c}$ is Boolean or there are no $\mathcal{I}_{k}$-inferences $J^{\prime},(t \leftarrow \mathfrak{c}) \vdash_{\mathcal{I}_{k}} L$ with $J^{\prime}, \bar{L} \subseteq J$.

When adding $t \leftarrow \mathfrak{c}$ to $J$, acceptability prevents repetitions (cf. Condition (i)) and contradictions: if $t \leftarrow \mathfrak{c}$ is Boolean, its flip should not be in $J$, preserving plausibility (cf. Condition (i)); if $t \leftarrow \mathfrak{c}$ is first-order, and therefore has no flip, so that plausibility does not apply, acceptability ensures that none of the consequences one inference step away has its flip in $J$ (cf. Condition (ii)).

Definition 4 (Relevance). A term is $\mathcal{T}_{k}$-relevant for an assignment $H$, if either (i) it occurs in $H$ and has a $\mathcal{T}_{k}$-public sort, or (ii) it is an equality $t_{1} \simeq_{s} t_{2}$ whose terms $t_{1}$ and $t_{2}$ occur in $H$ and whose sort $s \in S_{k}$ is not $\mathcal{T}_{k}$-public.

Relevance organizes the division of labor among modules. For instance in the assignment $\{x \leftarrow \sqrt{5}, f(x) \leftarrow \sqrt{2}, f(y) \leftarrow \sqrt{3}\}, x$ and $y$ of sort R are RA-relevant, not EUF-relevant, assuming $\mathrm{R}$ is not EUF-public, while $x \simeq_{\mathrm{R}} y$ is EUF-relevant, not RA-relevant. Each theory has a mechanism to fix and communicate equalities between terms of a known sort, such as $x$ and $y$ : EUF does it by assigning a truthvalue to $x \simeq_{\mathrm{R}} y$; RA does it by assigning values to $x$ and $y$.

\section{Examples of Theory Modules}

In this section we give theory modules for several theories. We may use $\perp$ to stand for the assignment $\left(x \simeq_{\text {prop }} x\right) \leftarrow$ false for an arbitrary variable $x$. For brevity, we omit equality symbols from signatures and equality inference from modules.

\subsection{A Module for Propositional Logic}

$\Sigma_{\text {Bool }}$ has only the sort prop and the symbols $\neg:$ prop $\rightarrow$ prop, and $\vee, \wedge$ : $($ prop $\times$ prop $) \rightarrow$ prop. Let Bool ${ }^{+}$be the trivial extension with only $\{$true, false $\}$as Bool $^{+}$-values. $\mathcal{I}_{\text {Bool }}$ features an evaluation inference rule that derives the truth value $\mathfrak{b}$ of formula $l$, given truth values $\mathfrak{b}_{1}, \ldots, \mathfrak{b}_{m}$ of subformulae $l_{1}, \ldots, l_{m}$, and then, from left to right, two rules for negation, two rules for conjunction elimination, and two rules for unit propagation:

$$
\begin{aligned}
& \neg l \vdash_{\text {Bool }} \bar{l} \quad \frac{l_{1} \leftarrow \mathfrak{b}_{1}, \ldots, l_{m} \leftarrow \mathfrak{b}_{m} \vdash_{\text {Bool }} l \leftarrow \mathfrak{b}}{l_{1} \vee \cdots \vee l_{m}} \vdash_{\text {Bool }} \overline{l_{i}} \quad \frac{l_{1} \vee \cdots \vee l_{m}}{l_{1} \wedge \cdots \wedge l_{m}},\left\{\overline{l_{j}} \mid j \neq i\right\} \vdash_{\text {Bool }} l_{i} \\
& \overline{\neg l} \vdash_{\text {Bool } l} l \quad l_{1} \wedge \cdots \wedge l_{m} \vdash_{\text {Bool }} l_{i} \quad \overline{l_{1} \wedge \cdots \wedge l_{m}},\left\{l_{j} \mid j \neq i\right\} \vdash_{\text {Bool }} \overline{l_{i}}
\end{aligned}
$$

where $1 \leq j, i \leq m$, and, for the first rule, $l$ must be in the closure of $l_{1}, \ldots, l_{m}$

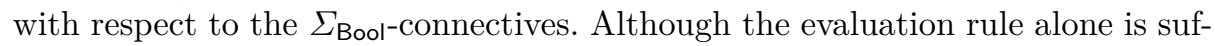
ficient for completeness (cf. Sect. 6.3), the other six rules, including in particular unit propagation as in CDCL, are obviously desirable. 


\subsection{A Theory Module for LRA}

Let $\Sigma_{\mathrm{LRA}}$ be as in Example 1 and $\mathrm{LRA}^{+}$be the extension that adds a constant $\tilde{q}$ and the axiom $\tilde{q} \simeq_{Q} q \cdot 1$ for each rational number $q \in \mathbb{Q}$. Here too, the first rule of $\mathcal{I}_{\text {LRA }}$ is an evaluation inference rule that derives the value $\mathfrak{b}$ of formula $l$, given values $\tilde{q_{1}}, \ldots, \tilde{q_{m}}$ of subterms $t_{1}, \ldots, t_{m}$ of sort $\mathrm{Q}$ :

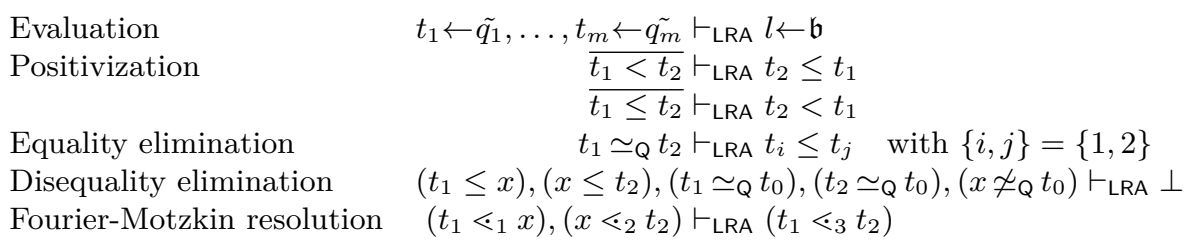

where $t_{0}, t_{1}, t_{2}$, and $x$ are terms of sort $\mathrm{Q} ; x$ is a $\Sigma_{\mathrm{LRA}}$-variable that is not free in $t_{0}, t_{1}, t_{2}$ (cf. Sect. 6 ); $\lessdot_{1}, \leftarrow_{2}, \leftarrow_{3} \in\{<, \leq\}$ and $\lessdot_{3}$ is $<$ if and only if either $\lessdot_{1}$ or $\lessdot_{2}$ is $<$. For the first rule, $l$ must be a formula whose normal form is in the closure of $t_{1}, \ldots, t_{m}$ with respect to the symbols of $F_{\text {LRA }}$. For example, $w-2 \simeq_{Q} w$ can be normalized to $-2 \simeq_{Q} 0$ and evaluates to false. In the last two rules, each formula $l$ appearing on the left stands for any formula that can be normalized to $l$. For instance, Fourier-Motzkin (FM) resolution applies to $y-x<2 \cdot y$ and $2 \cdot x<3$ yielding $-y<\frac{3}{2}$. The three linear combinations of constraints in the solution of problem $R$ in Sect. 1 are instances of FM resolution, as a linear combination $e_{1}+z<c_{1}, e_{2}-z<c_{2} \vdash e_{1}+e_{2}<c_{1}+c_{2}$ is expressed as an FM resolution $e_{2}-c_{2}<z, z<c_{1}-e_{1} \vdash_{\text {LRA }} e_{2}-c_{2}<c_{1}-e_{1}$.

\subsection{A Theory Module for EUF}

For a signature $\Sigma_{\text {EUF }}=\left(S, \simeq_{S} \cup F\right), \mathcal{I}_{\text {EUF }}$ may include

$$
\begin{aligned}
\left(t_{i} \simeq u_{i}\right)_{i=1 \ldots m}, f\left(t_{1}, \ldots, t_{m}\right) & \neq f\left(u_{1}, \ldots, u_{m}\right) \\
\left(t_{i} \simeq u_{i}\right)_{i=1 \ldots m} & \vdash_{\text {EUF }} f\left(t_{1}, \ldots, t_{m}\right) \simeq f\left(u_{1}, \ldots, u_{m}\right) \\
\left(t_{i} \simeq u_{i}\right)_{i=1 \ldots m, i \neq j}, f\left(t_{1}, \ldots, t_{m}\right) \nsucceq f\left(u_{1}, \ldots, u_{m}\right) & \vdash_{\text {EUF }} t_{j} \nsucceq u_{j}
\end{aligned}
$$

for all symbols $f \in F$. The first rule alone is sufficient for completeness: it captures a lazy approach that does not propagate anything before equalities between existing terms are found to be in contradiction with a congruence axiom [9]. The other two rules can be used directly for eager congruence propagation. Since $\mathcal{I}_{\text {EUF }}$ does not use first-order assignments, no sort needs to be EUF-public, and the only assignments assign truth values to equalities. Alternatively, one may make the sorts in $S$ EUF-public, with a countably infinite collection of EUF ${ }^{+}$-values in each sort and no axioms about them. Equality inferences can employ assignments of $\mathrm{EUF}^{+}$-values to determine whether terms are equal, using $\mathrm{EUF}^{+}$-values as identifiers for equivalence classes of terms. For example, assume that $\mathfrak{c}_{1}, \mathfrak{c}_{2}$, and $\mathfrak{c}_{3}$ are distinct EUF ${ }^{+}$-values. The assignment $\left\{x \leftarrow \mathfrak{c}_{1}, y \leftarrow \mathfrak{c}_{1}, f(x) \leftarrow \mathfrak{c}_{2}\right\}$ places $x$ and $y$ in the equivalence class $\mathfrak{c}_{1}$, and $f(x)$ in class $\mathfrak{c}_{2}$. If $f(y) \leftarrow \mathfrak{c}_{3}$ is added to the assignment, two equality inferences and an application of the first rule of $\mathcal{I}_{\text {EUF }}$ expose a conflict in the above-mentioned lazy style. 


\subsection{A Theory Module for Arrays}

The array sort constructor builds from an index sort $I$ and a value sort $V$ the sort $I \Rightarrow V$ of arrays with indices in $I$ and values in $V$. Consider a signature $\Sigma_{\text {Arr }}=(S, F)$, where $S$ is the free closure of a set of basic sorts with respect to the array sort constructor, and $F$ is

$$
\begin{array}{l|l}
\left\{\operatorname{select}_{I \Rightarrow V}:(I \Rightarrow V) \times I \rightarrow V\right. & (I \Rightarrow V) \in S\} \\
\cup\left\{\text { store }_{I \Rightarrow V}:(I \Rightarrow V) \times I \times V \rightarrow(I \Rightarrow V)\right. & (I \Rightarrow V) \in S\} \\
\cup\left\{\operatorname{diff}_{I \Rightarrow V}:(I \Rightarrow V) \times(I \Rightarrow V) \rightarrow I\right. & (I \Rightarrow V) \in S\},
\end{array}
$$

where $\operatorname{diff}_{I \Rightarrow V}$ is the Skolem function symbol that arises from clausifying the extensionality axiom for array sort $I \Rightarrow V$. For brevity, subscripts are omitted, $\operatorname{select}(a, i)$ is written as $a[i]$, and store $(a, i, v)$ as $a[i]:=v$. Module $\mathcal{I}_{\text {Arr }}$ features the following rules, where $a, b, c, d$ are variables of any $I \Rightarrow V$ sort, $u, v$ are variables of sort $V$, and $i, j, k$ of sort $I$ :

$$
\begin{aligned}
a \simeq b, i \simeq j, a[i] \nsucceq b[j] & \vdash_{\text {Arr }} \perp \\
a \simeq b, i \simeq j, u \simeq v,(a[i]:=u) \neq(b[j]:=v) & \vdash_{\text {Arr }} \perp \\
b \simeq(a[i]:=u), i \simeq j, b[j] \nsucceq u & \vdash_{\text {Arr }} \perp \\
b \simeq(a[i]:=u), i \neq j, j \simeq k, a[j] \nsucceq b[k] & \vdash_{\text {Arr }} \perp \\
a \neq b & \vdash_{\text {Arr }} a[\operatorname{diff}(a, b)] \nsucceq b[\operatorname{diff}(a, b)] \\
a \simeq c, b \simeq d, \operatorname{diff}(a, b) \neq \operatorname{diff}(c, d) & \vdash_{\text {Arr }} \perp
\end{aligned}
$$

The first two inference rules capture the congruence axioms for select and store. The third and fourth rules correspond to the read-over-write axioms. The fifth rule corresponds to the clausal form of the extensionality axiom; it is the only rule that can produce new terms. The last rule states the congruence axiom for diff. These rules are triggered by the truth-values of equalities. As with $\mathcal{I}_{\text {EUF }}$, in order to determine whether equalities hold, one has the option of declaring all sorts to be Arr-public, with infinitely many $\mathrm{Arr}^{+}$-values used as identifiers of equivalence classes. One can also add rules for eager propagations of equalities.

\subsection{Generic Theory Modules for Equality Sharing}

Assume $\mathcal{T}$ is a stably infinite theory with signature $\Sigma=(S, F)$ and equipped with a $\mathcal{T}$-satisfiability procedure. Its inference module $\mathcal{I}_{\mathcal{T}}$ comprises the rule

$$
l_{1} \leftarrow \mathfrak{b}_{1}, \ldots, l_{m} \leftarrow \mathfrak{b}_{m} \vdash_{\mathcal{T}} \perp
$$

that fires when the conjunction of the literals corresponding to the Boolean assignments on the left is $\mathcal{T}$-unsatisfiable. Unlike the previous ones, this module is coarse-grained, in the sense that a single application of its inference rule requires the execution of a $\mathcal{T}$-satisfiability procedure. As with EUF and Arr, one has the option of declaring non-Boolean sorts $\mathcal{T}$-public to determine equalities. If the $\mathcal{T}$-satisfiability procedure can produce unsatisfiable cores, we can restrict the above rule so that the assignment on the left is an unsatisfiable core. This provides a more precise conflict resolution mechanism, which leads us to Sect. 5 . 


\section{The CDSAT Inference System}

In this section we present CDSAT and exemplify its features by applying it to problems $R$ and $P$ in the introduction. A CDSAT derivation transforms a state consisting of a trail $\Gamma$. A trail is a sequence of distinct singleton assignments that are either justified assignments, denoted ${ }_{H \vdash} A$, or decisions, denoted ?A. The justification $H$ in $H \vdash A$ is a set of singleton assignments that appear before $A$ in the trail. For instance, a theory inference $J \vdash_{\mathcal{I}_{k}} L$ for some $k, 1 \leq k \leq n$, can justify adding $J \vdash L$ to the trail. A decision is written ? A because it is generally a guess. A trail can be used as an assignment by ignoring order and justifications.

\begin{tabular}{|cccr|}
\hline \multicolumn{3}{|c|}{ Phase 1 } \\
id & trail items & just lev \\
\hline $0-2 \cdot x-y<0$ & \{\} & 0 \\
1 & $x+y<0$ & \{\} & 0 \\
2 & $x<-1$ & \{\} & 0 \\
\hline 3 & $y \leftarrow 0$ & & 1 \\
4 & $-y<-2$ & $\{0,2\}$ & 0 \\
conflict $E^{1}:\{3,4\}$ & 1 \\
\hline
\end{tabular}

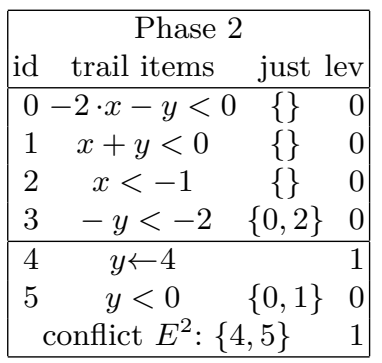

\begin{tabular}{|cccc|}
\hline \multicolumn{4}{|c|}{ Phase 3 } \\
id & trail items & just lev \\
\hline $0-2 \cdot x-y<0$ & \{\} & 0 \\
1 & $x+y<0$ & \{\} & 0 \\
2 & $x<-1$ & \{\} & 0 \\
3 & $-y<-2$ & $\{0,2\}$ & 0 \\
4 & $y<0$ & $\{0,1\}$ & 0 \\
\hline 5 & $0<-2$ & $\{3,4\}$ & 0 \\
& conflict $E^{3}:\{5\}$ & 0 \\
\hline
\end{tabular}

Fig. 2. CDSAT derivation in one theory (LRA)

The evolution of the trail for problem $R$ is described in three successive phases in Fig. 2. The input is shown above the horizontal line of Phase 1. In each proceeding phase, the assignments above the horizontal line are those inherited from the previous phase. Assignments are numbered in chronological order, and their numbers, shown in the first column, are used as identifiers. For every justified assignment, the justification is shown as a set of identifiers in the third column. In the sequel, $A_{n}^{m}$ is the assignment with identifier $n$ in phase $m$. For example, the justification of $A_{4}^{1}$ is $\{0,2\}$, because $A_{4}^{1}$ is derived by the FM resolution rule of $\mathcal{I}_{\text {LRA }}$ from $A_{0}^{1}$ and $A_{2}^{1}$. The last column shows the level of $A$ as defined next.

Definition 5 (Level). Given a trail $\Gamma$ with assignments $A_{0}, \ldots, A_{m}$,

$$
\text { level }_{\Gamma}\left(A_{i}\right)= \begin{cases}1+\max \left\{\operatorname{level}_{\Gamma}\left(A_{j}\right) \mid j<i\right\} & \text { if } A_{i} \text { is a decision, } \\ \operatorname{level}_{\Gamma}(H) & \text { if } A_{i} \text { has justification } H ;\end{cases}
$$

Given a $\mathcal{T}_{\infty}$-assignment $H \subseteq \Gamma$,

$$
\text { level }_{\Gamma}(H)= \begin{cases}0 & \text { if } H=\emptyset \\ \max \left\{\operatorname{level}_{\Gamma}(A) \mid A \in H\right\} & \text { otherwise. }\end{cases}
$$

The restriction of a trail $\Gamma$ to its elements of level at most $m$ is written $\Gamma^{\leq m}$.

The rules of the CDSAT inference system, given in Fig. 3, comprise search rules, whose application is denoted by $\longrightarrow$, and conflict resolution rules, whose application is denoted by $\Longrightarrow$, with transitive closure $\Longrightarrow$. The system is parameterized by a set $\mathcal{B}$ of terms, called global basis, used to limit the range of terms that CDSAT may generate, in order to ensure termination (cf. Sect. 6.2). 


\begin{tabular}{|c|c|}
\hline \multicolumn{2}{|l|}{ SEARCH RULES } \\
\hline Decide $\quad \Gamma \longrightarrow \Gamma, ? A$ & $\begin{array}{l}\text { if } A \text { is a } \mathcal{T}_{k} \text {-assignment for a } \mathcal{T}_{k} \text {-relevant term of } \Gamma \\
\text { that is acceptable for } \Gamma_{\mathcal{T}_{k}} \text { and } \mathcal{I}_{k} \text {, with } 1 \leq k \leq n\end{array}$ \\
\hline \multicolumn{2}{|c|}{$\begin{array}{l}\text { The next three rules share the conditions: } \\
J \subseteq \Gamma,\left(J \vdash_{\mathcal{I}_{k}} L\right) \text {, and } L \notin \Gamma \text {, for some } k, 1 \leq k \leq n\end{array}$} \\
\hline Deduce $\quad \Gamma \longrightarrow \Gamma,{ }_{J \vdash} L$ & if $\bar{L} \notin \Gamma$, and $\bar{L}$ is of the form $l \leftarrow \mathfrak{b}$ for some $l \in \mathcal{B}$ \\
\hline$\Gamma \longrightarrow$ unsat & if $\bar{L} \in \Gamma$ and level ${ }_{\Gamma}(J, \bar{L})=0$ \\
\hline ConflictSolve $\Gamma \longrightarrow \Gamma^{\prime}$ & if $\bar{L} \in \Gamma$, level $_{\Gamma}(J, \overline{\bar{L}})>0$, and $\langle\Gamma ; J, \bar{L}\rangle \Longrightarrow^{*} \Gamma^{\prime}$ \\
\hline \multicolumn{2}{|l|}{ CONFLICT RESOLUTION RULES } \\
\hline \multicolumn{2}{|l|}{ Undo } \\
\hline$\Longrightarrow \Gamma^{\leq m-1}$ & if $A$ is a first-order decision of level $m>\operatorname{level}_{\Gamma}(E)$ \\
\hline Backjump & \\
\hline $\begin{array}{l}\quad\langle\Gamma ; E, L\rangle \quad \Longrightarrow \Gamma^{\leq m}, E \vdash L \\
\text { Resolve }\end{array}$ & if level ${ }_{\Gamma}(L)>m$, where $m=\operatorname{level}_{\Gamma}(E)$ \\
\hline$\Longrightarrow\langle\Gamma ; E \cup H\rangle$ & $\begin{array}{l}\text { if } H \vdash A \text { is in } \Gamma \text { and } H \text { does not contain a } \\
\text { first-order decision whose level is level }{ }_{\Gamma}(E, A)\end{array}$ \\
\hline $\begin{array}{l}\text { UndoDecide } \\
\qquad\left\langle\Gamma ; E, L, L^{\prime}\right\rangle \Longrightarrow \Gamma^{\leq m-1}, ? \bar{L}\end{array}$ & $\begin{array}{l}\text { if } H \vdash L \text { and } H_{H^{\prime} \vdash} L^{\prime} \text { are in } \Gamma \text { and } H \cap H^{\prime} \text { contains a } \\
\text { first-order decision of level } m=\operatorname{level}_{\Gamma}\left(E, L, L^{\prime}\right)\end{array}$ \\
\hline
\end{tabular}

Fig. 3. The CDSAT inference system

The global basis is fixed throughout a CDSAT derivation but depends on the input problem. We describe next the CDSAT rules, beginning with the search rules. The Decide rule extends a trail $\Gamma$ with a theory assignment $A$ without justifying it by a theory inference: it is a decision. $A$ assigns a value to a relevant term, and is acceptable for the theory view of the trail and the theory module (cf. Definitions 2, 3 and 4). In Fig. 2, $y$ is the relevant term in $A_{3}^{1}$ and $A_{4}^{2}$.

The Deduce rule extends a trail $\Gamma$ with an assignment $L$ justified by a theory inference $J \vdash_{\mathcal{I}_{k}} L$. In Fig. 2, Deduce infers $A_{4}^{1}$ from $\left\{A_{0}^{1}, A_{2}^{1}\right\}, A_{5}^{2}$ from $\left\{A_{0}^{2}, A_{1}^{2}\right\}$, and $A_{5}^{3}$ from $\left\{A_{3}^{3}, A_{4}^{3}\right\}$, by using the FM resolution rule of $\mathcal{I}_{\text {LRA }}$.

Rules Fail and ConflictSolve apply to a trail $\Gamma$ that is conflicting because a theory inference $J \vdash_{\mathcal{I}_{k}} L$ contradicts an assignment $\bar{L}$ already present in $\Gamma$. The set $J \cup\{\bar{L}\}$ is the conflict. Its level is denoted level $\Gamma_{\Gamma}(J, \bar{L})$. If it is 0 , rule Fail returns unsat (e.g., $E^{3}$ in Fig. 2). If it is greater than 0 (e.g., $E^{1}$ and $E^{2}$ in Fig. 2), rule ConflictSolve triggers a series of conflict resolution steps transforming $\Gamma$ into a trail $\Gamma^{\prime}$ where the conflict is solved. In all three conflicts in Fig. 2, the inferences that expose the conflict are applications of the evaluation rule of $\mathcal{I}_{\text {LRA }}$ : $y \leftarrow 0 \vdash \overline{-y<-2}$ for $E^{1}, y \leftarrow 4 \vdash \overline{y<0}$ for $E^{2}$, and $\emptyset \vdash \overline{0<-2}$ for $E^{3}$.

We now describe the conflict resolution rules, referring to Fig. 2 and 4 for their application to problems $R$ and $P$, respectively. Conflict resolution rules operate on pairs $\langle\Gamma ; E\rangle$, where $\Gamma$ is a trail and $E$ is a set of assignments in $\Gamma$ termed conflict. If the conflict contains a first-order decision $A$, whose level $n$ is greater than that of the rest of the conflict, rule Undo removes $A$ and all assignments of level greater than or equal to $n$. In Fig. 2, rule Undo solves conflicts $E^{1}$ and $E^{2}$. 


\begin{tabular}{|rcrr|}
\hline \multicolumn{4}{c}{ Phase 1 } \\
id & trail items & just lev \\
\hline 0 & $f((a[i]:=v)[j]) \simeq w$ & \{\} & 0 \\
1 & $w-2 \simeq f(u)$ & \{\} & 0 \\
2 & $i \simeq j$ & \{\} & 0 \\
3 & $u \simeq v$ & \{\} & 0 \\
\hline 4 & $u \leftarrow \mathfrak{c}$ & 1 \\
5 & $v \leftarrow \mathfrak{c}$ & 2 \\
6 & $(a[i]:=v)[j] \leftarrow \mathfrak{c}$ & 3 \\
7 & $w \leftarrow 0$ & 4 \\
8 & $f((a[i]:=v)[j]) \leftarrow 0$ & & 5 \\
9 & $f(u) \leftarrow-2$ & 6 \\
10 & $u \simeq(a[i]:=v)[j]$ & $\{4,6\}$ & 3 \\
$11 f(u) \neq f((a[i]:=v)[j])\{8,9\}$ & 6 \\
\multicolumn{3}{c}{ conflict $E^{1}:\{10,11\}$} & 6 \\
\hline \multicolumn{3}{|c}{}
\end{tabular}

\begin{tabular}{|c|c|c|c|}
\hline \multicolumn{4}{|c|}{ Phase 3} \\
\hline id & trail items & just 1 & lev \\
\hline 0 . & $((a[i]:=v)[j]) \simeq w$ & \{\} & 0 \\
\hline 1 & $w-2 \simeq f(u)$ & \{\} & 0 \\
\hline 2 & $i \simeq j$ & \{\} & 0 \\
\hline 3 & $u \simeq v$ & \{\} & 0 \\
\hline 4 & $u \not ॅ(a[i]:=v)[j]$ & $\{0,1\}$ & 0 \\
\hline 5 & $u \leftarrow \mathfrak{c}$ & & 1 \\
\hline 6 & $v \leftarrow \mathfrak{c}$ & & 2 \\
\hline 7 & $(a[i]:=v)[j] \leftarrow \mathfrak{d}$ & & 3 \\
\hline 8 & $v \not \chi(a[i]:=v)[j]$ & $\{6,7\}$ & $\begin{array}{l}3 \\
2\end{array}$ \\
\hline & conflict $E^{\mathrm{u}}:\{2,8\}$ & & 3 \\
\hline
\end{tabular}

\begin{tabular}{|rcrr|}
\hline \multicolumn{4}{|c|}{ Phase 2} \\
id & trail items & just lev \\
\hline 0 & $f((a[i]:=v)[j]) \simeq w$ & \{\} & 0 \\
1 & $w-2 \simeq f(u)$ & \{\} & 0 \\
2 & $i \simeq j$ & \{\} & 0 \\
3 & $u \simeq v$ & \{\} & 0 \\
4 & $u \leftarrow \mathfrak{c}$ & & 1 \\
5 & $v \leftarrow \mathfrak{c}$ & & 2 \\
6 & $(a[i]:=v)[j] \leftarrow \mathfrak{c}$ & & 3 \\
7 & $u \simeq(a[i]:=v)[j]$ & $\{4,6\}$ & 3 \\
\hline $8 f(u) \simeq f((a[i]:=v)[j])$ & $\{7\}$ & 3 \\
9 & $f(u) \simeq w$ & $\{0,8\}$ & 3 \\
10 & $w-2 \simeq w$ & $\{1,9\}$ & 3 \\
& conflict $E_{1}^{2}:\{10\}$ & & 3 \\
& conflict $E_{2}^{2}:\{1,9\}$ & 3 \\
& conflict $E_{3}^{2}:\{0,1,8\}$ & 3 \\
& conflict $E_{4}^{2}:\{0,1,7\}$ & 3 \\
\hline \multicolumn{4}{|c}{} \\
\hline
\end{tabular}

\begin{tabular}{|cccc|}
\hline \multicolumn{4}{|c|}{ Phase 4} \\
id & trail items & just lev \\
\hline 0 & $f((a[i]:=v)[j]) \simeq w$ & \{\} & 0 \\
1 & $w-2 \simeq f(u)$ & \{\} & 0 \\
2 & $i \simeq j$ & \{\} & 0 \\
3 & $u \simeq v$ & \{\} & 0 \\
4 & $u \neq(a[i]:=v)[j]$ & $\{0,1\}$ & 0 \\
\hline 5 & $v \simeq(a[i]:=v)[j]$ & $\{2\}$ & 0 \\
& conflict $E^{4}:\{3,4,5\}$ & 0 \\
\hline
\end{tabular}

Fig. 4. CDSAT derivation in three theories (LRA, EUF, and Arr)

The Backjump rule is similar in that the conflict contains an assignment whose level is greater than that of all others. Backjump applies if this assignment is a Boolean assignment $L$; its flip $\bar{L}$ is justified by the rest of the conflict $E$. Therefore we backjump to the level of $E$, and add ${ }_{E \vdash} \bar{L}$ to the trail. Assignment $\bar{L}$ is a Unique Implication Point [14]. We see an application of this rule in Fig. 4. Phase 1 starts with a series of decisions, from $A_{4}^{1}$ through $A_{9}^{1}$, where $\mathfrak{c}$ is an Arr ${ }^{+}$-value of sort $V$, and $A_{5}^{1}$ is the only acceptable choice given $A_{3}^{1}$ and $A_{4}^{1}$. Then Deduce generates $A_{10}^{1}$ and $A_{11}^{1}$ by equality inferences (cf. Fig. 1), and ConflictSolve applies, as $E^{1} \vdash_{\mathcal{I}_{\text {EUF }}} \perp$ by the first inference rule of $\mathcal{I}_{\text {EUF. }}$. Rule Undo does not apply to $E^{1}$, because $E^{1}$ does not contain first-order decisions, but rule Backjump does apply, with $A_{11}^{1}$ playing the role of $L$. CDSAT jumps back to level 3, the level of $A_{10}^{1}$, and places $\overline{A_{11}^{1}}$ on the trail with justification $A_{10}^{1}$, named $A_{7}^{2}$ in Phase 2. Deduce places $A_{9}^{2}$ and $A_{10}^{2}$ on the trail by transitivity of equality (cf. Fig. 1). ConflictSolve applies as $\emptyset \vdash_{\text {LRA }} w-2 \nsucceq w$. 
The Resolve rule unfolds a conflict by replacing an assignment $A$ in the conflict with its justification $H$, provided $H$ does not introduce a first-order decision of the same level as that of the conflict. Starting from $E_{1}^{2}$ in Fig. 4, three Resolve steps yield conflict $E_{4}^{2}$. Resolve does not apply to $A_{7}^{2}$ because its justification contains $A_{6}^{2}$. Backjump solves $E_{4}^{2}$ by jumping back to level 0 and flipping $A_{7}^{2}$ into $A_{4}^{3}$. Then CDSAT guesses $A_{5}^{3}$ through $A_{7}^{3}$, where $A_{6}^{3}$ is forced by $A_{3}^{3}$ and $A_{5}^{3}$. For $A_{7}^{3}$, another $A^{+}{ }^{+}$-value $\mathfrak{d}$ of sort $V$ is used, since $A_{4}^{3}$ and $A_{5}^{3}$ prevent assigning $\mathfrak{c}$ to $(a[i]:=v)[j]$. Deduce generates $A_{8}^{3}$ by an equality inference, and conflict $E^{3}$ arises as $(i \simeq j),(v \not(a[i]:=v)[j]) \vdash$ Arr $\perp$. Backjump solves $E^{3}$ by jumping back to level 0 and flipping $A_{8}^{3}$ into $A_{5}^{4}$. The final conflict $E^{4}$ violates transitivity of equality, and because $E^{4}$ is at level 0, rule Fail closes the derivation.

The UndoDecide rule corresponds to $T$ backjump-decide [5] and semantic split [9]. It applies when the conflict contains two assignments $L$ and $L^{\prime}$ whose justifications include a first-order decision of maximal level in the conflict. Rule Resolve is barred from replacing $L$ or $L^{\prime}$ by their justification, so the only way to solve the conflict is to trade the first-order decision for a Boolean decision on the flip of $L$ or $L^{\prime}$. In Fig. 5, conflict $E_{4}$ is solved by UndoDecide, as both $A_{3}^{1}$ and $A_{4}^{1}$ are justified by the first-order decision $A_{2}^{1}$. UndoDecide arbitrarily chooses to flip $A_{3}^{1}$, and then values can be found for variables without raising a conflict: the problem is satisfiable.

\begin{tabular}{|cccr|}
\hline \multicolumn{4}{|c|}{ Phase 1 } \\
id & trail items & just lev \\
\hline 0 & $(x>1) \vee(y<0)$ & \{\} & 0 \\
$1(x<-1) \vee(y>0)$ & \{\} & 0 \\
\hline 2 & $x \leftarrow 0$ & & 1 \\
3 & $\frac{x>1}{x<-1}$ & $\{2\}$ & 1 \\
4 & $y<\}$ & 1 \\
5 & $y<0$ & $\{0,3\}$ & 1 \\
6 & $y>0$ & $\{1,4\}$ & 1 \\
7 & $0<0$ & $\{5,6\}$ & 1 \\
& conflict $E_{1}:\{7\}$ & 1 \\
& conflict $E_{2}:\{5,6\}$ & 1 \\
conflict $E_{3}:\{0,3,6\}$ & 1 \\
conflict $E_{4}:\{0,1,3,4\}$ & 1 \\
\hline
\end{tabular}

\begin{tabular}{|cccr|}
\hline \multicolumn{4}{|c|}{ Phase 2} \\
id & trail items & just & lev \\
\hline 0 & $(x>1) \vee(y<0)$ & \{\} & 0 \\
$1(x<-1) \vee(y>0)$ & \{\} & 0 \\
\hline 2 & $x>1$ & & 1 \\
3 & $x \leftarrow 2$ & & 2 \\
4 & $x<-1$ & $\{3\}$ & 2 \\
5 & $y>0$ & $\{1,4\}$ & 2 \\
6 & $y \leftarrow 1$ & & 3 \\
7 & $\overline{y<0}$ & $\{6\}$ & 3 \\
\hline
\end{tabular}

Fig. 5. CDSAT derivation in two theories (Bool and LRA)

\section{Soundness, Termination, and Completeness of CDSAT}

In this section we establish soundness, termination, and completeness of CDSAT. The proofs of these theorems can be found in the technical report [3]. The key point is to reduce such global properties to theory-local requirements for the theory modules involved in the combination. In other words, we need to discover sufficient conditions whose fulfillment by all theory modules $\mathcal{I}_{1}, \ldots, \mathcal{I}_{n}$ ensures soundness, termination, and completeness of the combined system.

This reduction raises the issue of how to handle the fact that assignments contain symbols unknown to a theory. For the combination of theory modules to be truly modular, $\mathcal{I}_{k}$ treats as a variable any subterm whose root is a symbol foreign to $\mathcal{T}_{k}$. Formally, if $\Sigma=(S, F)$ is a signature included in $\Sigma_{\infty}$, the free $\Sigma$ variables $\mathrm{fv}_{\Sigma}(t)$ of a term $t$ are the maximal subterms of $t$, in the subterm ordering $\triangleleft$, whose root is not in $F$. For a set $X$ of terms, $\mathrm{fv}_{\Sigma}(X)=\left\{u \mid u \in \mathrm{fv}_{\Sigma}(t), t \in X\right\}$, 
and for an assignment $H, \mathrm{fv}_{\Sigma}(H)=\left\{u \mid u \in \mathrm{fv}_{\Sigma}(t), t \leftarrow \mathfrak{c} \in H\right\}$. For problem $P$ in Sect. 1, signatures $\Sigma_{\mathrm{LRA}}, \Sigma_{\mathrm{EUF}}, \Sigma_{\text {Arr }}$ of Example 1 define for instance:

$$
\begin{aligned}
& \mathrm{fv}_{\Sigma_{\mathrm{LRA}}}(P)= \\
& \mathrm{fv}_{\Sigma_{\mathrm{EUF}}}(P)=\{(\operatorname{select}(\operatorname{store}(a, i, v), j)), w, f(u), i \simeq j, u \simeq v\} \\
& \mathrm{fv}_{\Sigma_{\text {Arr }}}(P)=\{f(\operatorname{select}(\operatorname{store}(a, i, v), j), w, u, w-2, i \simeq j, v\} \\
&
\end{aligned}
$$

In the next two definitions, $\mathcal{T}$ and $\Sigma$ stand for either $\mathcal{T}_{\infty}$ and $\Sigma_{\infty}$ or $\mathcal{T}_{k}$ and $\Sigma_{k}, 1 \leq k \leq n$. The identification of sufficient conditions for soundness and completeness and their proofs demand that we relate the assignments manipulated by CDSAT to models. This is the purpose of the notion of endorsement:

Definition 6 (Endorsement). $A \mathcal{T}^{+}[\mathcal{V}]$-model $\mathcal{M}$ endorses a $\mathcal{T}$-assignment $J$, such that $\mathrm{fv}_{\Sigma}(J) \subseteq \mathcal{V}$, if for all $t \leftarrow \mathfrak{c}$ in $J, \mathcal{M}(t)=\mathfrak{c}^{\mathcal{M}}$.

For Boolean assignments, it means that formulae are interpreted with the correct truth values. Definition 6 uses $\mathcal{T}^{+}$-models, because assignments contain $\mathcal{T}^{+}$-values (e.g., $\sqrt{2}$ ), and therefore we need models that interpret $\mathcal{T}^{+}$-values, and interpret them consistently with the axioms (e.g., $\sqrt{2} \cdot \sqrt{2}=2$ ).

Definition 7 (View endorsement). $A \mathcal{T}^{+}[\mathcal{V}]$-model $\mathcal{M}$ view-endorses $a \mathcal{T}_{\infty}$ assignment $H$ with $\mathrm{fv}_{\Sigma}(H) \subseteq \mathcal{V}$, if it endorses its $\mathcal{T}$-view $H_{\mathcal{T}}$.

This definition combines endorsement and view (cf. Definition 2) because CDSAT works with $\mathcal{T}_{\infty}$-assignments, which mix $\mathcal{T}_{k}$-assignments for any $k, 1 \leq k \leq n$. If $H$ is Boolean, view endorsement collapses to endorsement.

\subsection{Soundness}

The sufficient condition for soundness is that for every theory module $\mathcal{I}_{k}$, for all $\mathcal{I}_{k}$-inferences $J \vdash_{\mathcal{I}_{k}} L$, and all $\mathcal{V}$ such that $\mathrm{fv}_{\Sigma_{k}}(J \cup\{L\}) \subseteq \mathcal{V}$, every $\mathcal{T}_{k}^{+}[\mathcal{V}]$ model that view-endorses $J$ endorses $L$. Under this assumption, we prove that CDSAT is sound, by showing that each transition rule produces a trail whose restriction to level 0 is equisatisfiable to the input assignment.

Theorem 1 (Soundness). For all input assignments $H$, if a CDSAT derivation from $H$ reaches state unsat, no $\mathcal{T}_{\infty}^{+}[\mathcal{V}]$-model with $\mathrm{fv}_{\Sigma_{\infty}}(H) \subseteq \mathcal{V}$ viewendorses $H$; if $H$ is Boolean, no $\mathcal{T}_{\infty}[\mathcal{V}]$-model with $\mathrm{fv}_{\Sigma_{\infty}}(H) \subseteq \mathcal{V}$ endorses $H$.

All theory modules in Sect. 4 satisfy the soundness requirement.

\subsection{Termination}

As CDSAT allows the introduction of terms that are not in the input problem (cf. Deduce), termination is imperiled. For instance, applying the FM resolution rule of $\mathcal{I}_{\text {LRA }}$ to problem $R$ from Sect. 1, one can infer the formulae of Fig. 6. Such divergence is prevented by imposing finiteness of the global basis $\mathcal{B}$, that is the source of new terms in a CDSAT derivation.

$$
\begin{aligned}
& l_{0}:-2 \cdot x-y<0 \\
& l_{1}: \quad x+y<0 \\
& l_{2}: \quad x<-1 \\
& l_{3}: \quad-y<-2 \quad\left(l_{0}+2 l_{2}\right) \\
& l_{4}: \quad x<-2 \quad\left(l_{1}+l_{3}\right) \\
& l_{5}: \quad-y<-4 \quad\left(l_{0}+2 l_{4}\right) \\
& l_{6}: \quad x<-4 \quad\left(l_{1}+l_{5}\right) \\
& l_{7}: \quad-y<-8 \quad\left(l_{0}+2 l_{6}\right) \\
& \text { ‥ }
\end{aligned}
$$

Fig. 6. Divergence 
Theorem 2 (Termination). If the global basis $\mathcal{B}$ is finite, every CDSAT derivation is guaranteed to terminate.

Then the issue is to give sufficient conditions for the existence of a global basis $\mathcal{B}$, that is finite, and yet sufficiently rich for CDSAT to be complete. To address this question at the combination level we begin by imposing a similar requirement at the single theory level. We require that each theory module comes with a function basis $_{k}$, called local basis, that maps any finite set $X$ of terms to a finite set of terms $\operatorname{basis}_{k}(X)$, and has the following properties: it is (i) extensive

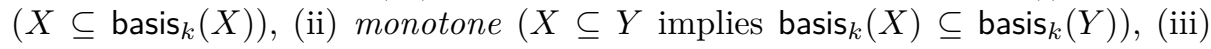
idempotent $\left(\operatorname{basis}_{k}\left(\operatorname{basis}_{k}(X)\right)=\operatorname{basis}_{k}(X)\right)$, (iv) downward-closed with respect to the subterm ordering (if $t \triangleleft u$ and $u \in \operatorname{basis}_{k}(X)$ then $t \in \operatorname{basis}_{k}(X)$ ), (v) closed with respect to equality (if $t, u \in$ basis $_{k}(X)$, of a sort $s$ different from prop, then $\left.\left(t \simeq_{s} u\right) \in \operatorname{basis}_{k}(X)\right)$, and (vi) does not introduce foreign symbols $\left(\mathrm{fv}_{\Sigma_{k}}\left(\operatorname{basis}_{k}(X)\right) \subseteq \mathrm{fv}_{\Sigma_{k}}(X) \cup \mathcal{V}_{\infty}\right)$.

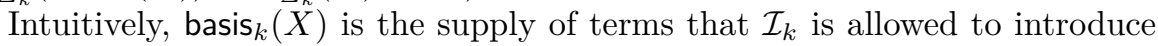
during a derivation from an input problem whose terms are in $X$. However, basis $_{k}(X)$ is not pre-computed. Furthermore, basis $_{k}$ should provide enough terms to make $\mathcal{I}_{k}$ complete, according to a notion of completeness of a theory module defined in the sequel in terms of both $\mathcal{I}_{k}$-inferences and basis $k$.

The divergence in Fig. 6 involves only $\mathcal{I}_{\text {LRA }}$. It can be avoided by assuming a fixed arbitrary order $\prec$ on $\Sigma_{\text {LRA-variables [9], and defining basis }}$ LRA as the function that saturates its argument with the terms introduced by all positivization inferences and by the FM resolution inferences $\left(t_{1} \lessdot_{1} x\right),\left(x \lessdot_{2} t_{2}\right) \vdash_{\text {LRA }}\left(t_{1} \lessdot_{3} t_{2}\right)$

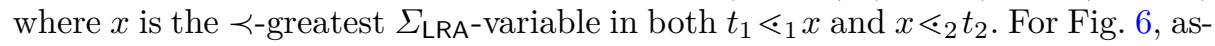
sume that $y \prec x$. Then $l_{3}$, generated by $(-y<2 \cdot x),(2 \cdot x<-2) \vdash_{\text {LRA }}(-y<-2)$, is in the local basis, whereas $l_{4}$, generated by $(x<-y),(-y<-2) \vdash$ LRA $(x<-2)$, is not, so that the series of inferences halts.

\subsection{Completeness}

As theory modules are used to extend the trail and reveal conflicts, the aim for completeness is that whenever no theory module can extend the trail, then the trail provides enough information to build a $\mathcal{T}_{\infty}^{+}$-model of the input problem. We begin by formalizing the concept that a theory module can extend an assignment.

Definition 8 (Assignment extension). Module $\mathcal{I}_{k}$ with local basis basis $_{k}$ can extend a $\mathcal{T}_{k}$-assignment $J$ if

- Either there exists a $\mathcal{T}_{k}$-assignment $t \leftarrow \mathfrak{c}$, for a $\mathcal{T}_{k}$-relevant term $t$ of $J$, that is acceptable for $J$ and $\mathcal{I}_{k}$;

- Or there exist a $\mathcal{T}_{k}$-assignment $J^{\prime} \subseteq J$, a formula $l \in \operatorname{basis}_{k}(J)$, and an $\mathcal{I}_{k}$-inference $J^{\prime} \vdash_{\mathcal{I}_{k}}(l \leftarrow \mathfrak{b})$ such that $(l \leftarrow \mathfrak{b}) \notin J$.

The first case is used for a Decide step and the second one for a Deduce, Fail, or ConflictSolve step. A module $\mathcal{I}_{k}$ is said to be complete, if for all plausible $\mathcal{T}_{k^{-}}$ assignments $J$ that $\mathcal{I}_{k}$ cannot extend, there exists a $\mathcal{T}_{k}^{+}\left[\mathrm{fv}_{\Sigma_{k}}(J)\right]$-model $\mathcal{M}$ that 
view-endorses $J$. However, when no theory module can extend its view of a trail $\Gamma$, the existence of a theory-specific model for $\Gamma$ for each theory does not imply the existence of a model for the combination of the theories. As in the equalitysharing method [16], these models need to agree on equalities between shared variables and on cardinalities of shared sorts. If all theories are stably infinite, the common cardinality is countably infinite. Nonetheless, there are interesting combinations that involve finite cardinalities, such as combining a theory with finite sorts and the theory of arrays with extensionality. CDSAT can handle such cases, if one of the combined theories, say $\mathcal{T}_{1}$, knows all the sorts (i.e., $S_{1}=S_{\infty}$ ) and offers information about their cardinalities. A combination of stably infinite theories is the instance of this scheme where $\mathcal{T}_{1}$ is a theory $\mathcal{T}_{\mathbb{N}}$ whose models interpret every sort in $S_{\infty} \backslash\{$ prop $\}$ as a countably infinite set.

The theory-specific requirements for completeness of CDSAT are that $\mathcal{I}_{1}$ is complete, and all other modules are complete relative to $\mathcal{T}_{1}$. The latter notion, that we call $\mathcal{T}_{1}$-completeness, in turn relies on $\mathcal{T}_{1}$-compatibility, defined below.

Definition 9 ( $\mathcal{T}_{1}$-compatibility). $A \mathcal{T}_{k}$-assignment $J$ is $\mathcal{T}_{1}$-compatible with $\mathcal{T}_{k}^{+}$, sharing a set of terms $G$, if for any $\mathcal{T}_{1}^{+}\left[\mathrm{fv}_{\Sigma_{1}}(J \cup G)\right]$-model $\mathcal{M}_{1}$ that viewendorses $J$, there exists a $\mathcal{T}_{k}^{+}\left[\mathrm{fv}_{\Sigma_{k}}(J \cup G)\right]$-model $\mathcal{M}$ that view-endorses $J$, such that for all sorts $s \in S_{k},\left|s^{\mathcal{M}}\right|=\left|s^{\mathcal{M}_{1}}\right|$, and for all terms $t$ and $t^{\prime}$ in $G$ of sort $s$, $\mathcal{M}(t)=\mathcal{M}\left(t^{\prime}\right)$ if and only if $\mathcal{M}_{1}(t)=\mathcal{M}_{1}\left(t^{\prime}\right)$.

A module $\mathcal{I}_{k}$ is $\mathcal{T}_{1}$-complete, if for all plausible $\mathcal{T}_{k}$-assignments $J$ that $\mathcal{I}_{k}$ cannot extend, $J$ is $\mathcal{T}_{1}$-compatible with $\mathcal{T}_{k}^{+}$, sharing all terms that occur in $J$. Then, the global basis $\mathcal{B}$ is stable, if $\operatorname{basis}_{k}(\mathcal{B}) \subseteq \mathcal{B}$ holds for all $k, 1 \leq k \leq n$.

Theorem 3 (Completeness). For all input assignments $H$, if the global basis $\mathcal{B}$ is stable and contains all terms that occur in $H$, whenever a CDSAT derivation from $H$ reaches a state $\Gamma$ other than unsat such that no CDSAT inference applies, there exists a $\mathcal{T}_{\infty}^{+}\left[\mathrm{fv}_{\Sigma_{\infty}}(\Gamma)\right]$-model that view-endorses $\Gamma$ and $H$ contained in $\Gamma$.

The proof of this theorem [3] relies on the following:

Lemma 1 (Model glueing). Let $H$ be an assignment and $G$ be the collection of shared terms inductively defined by:

$\frac{(t \leftarrow \mathfrak{c}) \in H}{t \in G} \quad \frac{u, u^{\prime} \in G \quad t \in \mathrm{fv}_{\Sigma_{i}}(u) \cap \mathrm{fv}_{\Sigma_{j}}\left(u^{\prime}\right) \quad i \neq j}{t \in G} \quad \frac{u \in G \quad t \in \mathrm{fv}_{\Sigma_{k}}(u) \backslash \mathcal{V}_{\infty}}{t \in G}$

If there exists a $\mathcal{T}_{1}^{+}\left[\mathrm{fv}_{\Sigma_{1}}(H)\right]$-model that view-endorses $H$, and such that for all $k, 2 \leq k \leq n$, the $\mathcal{T}_{k}$-view $H_{\mathcal{T}_{k}}$ is $\mathcal{T}_{1}$-compatible with $\mathcal{T}_{k}^{+}$sharing $G$, then there exists a $\mathcal{T}_{\infty}^{+}[\mathrm{fv}(H)]$-model that view-endorses $H$.

A derivation can reach a state satisfying the hypotheses of Lemma 1 long before it reaches a state that no module can extend. An implementation of a module could notify the main algorithm when the trail becomes $\mathcal{T}_{1}$-compatible with its theory. In this sense, Theorem 3 covers the worst-case scenario. A stable global basis can be obtained by taking $\mathcal{B}=\operatorname{basis}_{\pi(k)}\left(\ldots \operatorname{basis}_{\pi(1)}(X)\right)$, where $X$ is the set of terms occurring in the input assignment and $\pi$ is a 
permutation of $\{1, \ldots, k\}$ that satisfies the following property: if $i<j$ then basis $_{\pi(i)}\left(\operatorname{basis}_{\pi(j)}(X)\right) \subseteq$ basis $_{\pi(j)}\left(\right.$ basis $\left._{\pi(i)}(X)\right)$. A syntactic criterion on the local bases implies this permutability property [3].

For all theory modules of Sect. 4, except $\mathcal{I}_{\text {LRA }}$, we can define a local basis that makes them $\mathcal{T}_{\mathbb{N}}$-complete (cf. [3] for stronger completeness properties). For $\mathcal{I}_{\text {LRA }}$, the local basis basis LRA given above makes the module $\mathcal{T}_{\mathbb{N}}$-complete only under the strategy that assigns $\Sigma_{\mathrm{LRA}}$-variables in $\prec$-increasing order. Otherwise, considering again problem $R$ and the ordering $y \prec x$, the LRA-assignment $l_{0}, l_{1}, l_{2}, l_{3},(x \leftarrow 0)$ cannot be extended by $\mathcal{I}_{\text {LRA }}$ even though it is LRA-unsatisfiable. Indeed, the obvious FM resolution combining $l_{1}$ and $l_{3}$ would eliminate $y$, which

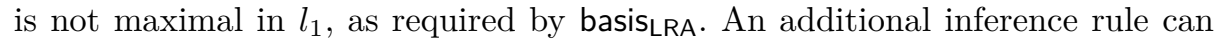
be added to $\mathcal{I}_{\text {LRA }}$ to make it complete regardless of strategy [3].

\section{Discussion}

In this paper we introduced CDSAT, a conflict-driven system for deciding the satisfiability of quantifier-free problems in the union of disjoint theories. CDSAT combines theory inference systems, termed theory modules. We presented several theory modules, including one for arrays which is the first integration of this theory in a conflict-driven combination. CDSAT lifts CDCL to SMT in the sense of satisfiability modulo multiple theories. Since it accepts input problems containing Boolean and first-order assignments, CDSAT solves a class of problems that extends SMT and that we call SMA for Satisfiability Modulo Assignments. For such problems, the input format presupposes the theory extensions (cf. Sect. 3).

CDSAT generalizes MCSAT $[5,8,9,19]$ to theory combinations. Furthermore, CDSAT solves the hitherto open problem of integrating conflict-driven procedures and the black-box solvers used in the equality sharing method [16]. CDSAT generalizes equality sharing itself, which corresponds to the case where all theories are stably infinite, all theory modules are black-boxes (cf. Sect. 4.5), and CDSAT decisions are limited to equalities between shared variables.

Clause learning, including theory lemmas, can be easily added to the version of CDSAT presented here [3]. Directions for future work include: the generation of proofs, by composition of theory inferences; efficient techniques to detect the applicability of theory inference rules and determine whether an assignment is acceptable (e.g., watched variables [9]); and heuristic strategies to make decisions and prioritize theory inferences.

Acknowledgments The authors thank Dejan Jovanović for fruitful discussions. Part of this research was conducted while the first author was an international fellow at the Computer Science Laboratory of SRI International, whose support is greatly appreciated. This research was funded in part by NSF grants 1528153 and CNS-0917375, by DARPA under agreement number FA8750-16-C-0043, and by grant "Ricerca di base 2015" of the Università degli Studi di Verona. The views and conclusions contained herein are those of the authors and should not be interpreted as necessarily representing the official policies or endorsements, either expressed or implied, of NSF, DARPA, or the U.S. Government. 


\section{References}

1. Barrett, C., Nieuwenhuis, R., Oliveras, A., Tinelli, C.: Splitting on demand in SAT modulo theories. In: Hermann, M., Voronkov, A. (eds.) Proceedings of the Thirteenth International Conference on Logic for Programming, Artificial Intelligence, and Reasoning (LPAR). Lecture Notes in Artificial Intelligence, vol. 4246, pp. 512-526. Springer (2006) 2

2. Bonacina, M.P.: On conflict-driven reasoning. In: Dutertre, B., Shankar, N. (eds.) Proceedings of the Sixth Workshop on Automated Formal Methods (AFM), at the Ninth NASA Formal Methods Symposium (NFM). pp. 1-9. To appear (2017), http://fm.csl.sri.com/AFM17/ 1

3. Bonacina, M.P., Graham-Lengrand, S., Shankar, N.: A model-constructing framework for theory combination. Tech. Rep. 99/2016, Dipartimento di Informatica, Università degli Studi di Verona, Verona, Italy, EU (November 2016), https: //hal.archives-ouvertes.fr/hal-01425305, also Technical Report of SRI International and INRIA - CNRS - École Polytechnique; revised April 2017 12, 15, 16

4. Cotton, S.: Natural domain SMT: A preliminary assessment. In: Chatterjee, K., Henzinger, T.A. (eds.) Proceedings of the Eighth International Conference on Formal Modeling and Analysis of Timed Systems (FORMATS). Lecture Notes in Computer Science, vol. 6246, pp. 77-91. Springer (2010) 1

5. de Moura, L., Jovanović, D.: A model-constructing satisfiability calculus. In: Giacobazzi, R., Berdine, J., Mastroeni, I. (eds.) Proceedings of the Fourteenth International Conference on Verification, Model Checking and Abstract Interpretation (VMCAI). Lecture Notes in Computer Science, vol. 7737, pp. 1-12. Springer (2013) $2,12,16$

6. Ganzinger, H., Rueß, H., Shankar, N.: Modularity and refinement in inference systems. Tech. Rep. CSL-SRI-04-02, Computer Science Laboratory, SRI International, Menlo Park, CA, USA (2004) 3

7. Haller, L., Griggio, A., Brain, M., Kroening, D.: Deciding floating-point logic with systematic abstraction. In: Cabodi, G., Singh, S. (eds.) Proceedings of the Twelfth International Conference on Formal Methods in Computer Aided Design (FMCAD). ACM and IEEE (2012) 1

8. Jovanović, D.: Solving nonlinear integer arithmetic with MCSAT. In: Bouajjani, A., Monniaux, D. (eds.) Proceedings of the Eighteenth International Conference on Verification, Model Checking and Abstract Interpretation (VMCAI). Lecture Notes in Computer Science, vol. 10145, pp. 330-346. Springer (2017) 2, 16

9. Jovanović, D., Barrett, C., de Moura, L.: The design and implementation of the model-constructing satisfiability calculus. In: Jobstman, B., Ray, S. (eds.) Proceedings of the Thirteenth Conference on Formal Methods in Computer Aided Design (FMCAD). ACM and IEEE (2013) 2, 5, 7, 12, 14, 16

10. Jovanović, D., de Moura, L.: Cutting to the chase: solving linear integer arithmetic. In: Bjørner, N., Sofronie-Stokkermans, V. (eds.) Proceedings of the Twenty-Third International Conference on Automated Deduction (CADE). Lecture Notes in Artificial Intelligence, vol. 6803, pp. 338-353. Springer (2011) 1

11. Jovanović, D., de Moura, L.: Solving non-linear arithmetic. In: Gramlich, B., Miller, D., Sattler, U. (eds.) Proceedings of the Sixth International Joint Conference on Automated Reasoning (IJCAR). Lecture Notes in Artificial Intelligence, vol. 7364, pp. 339-354. Springer (2012) 1 
12. Korovin, K., Tsiskaridze, N., Voronkov, A.: Conflict resolution. In: Gent, I.P. (ed.) Proceedings of the Fifteenth International Conference on Principles and Practice of Constraint Programming (CP). Lecture Notes in Computer Science, vol. 5732, pp. 509-523. Springer (2009) 1

13. Krstić, S., Goel, A.: Architecting solvers for SAT modulo theories: Nelson-Oppen with DPLL. In: Wolter, F. (ed.) Proceedings of the Sixth International Symposium on Frontiers of Combining Systems (FroCoS). Lecture Notes in Artificial Intelligence, vol. 4720, pp. 1-27. Springer (2007) 2

14. Marques Silva, J., Lynce, I., Malik, S.: Conflict-driven clause learning SAT solvers. In: Biere, A., Heule, M., Van Maaren, H., Walsh, T. (eds.) Handbook of Satisfiability, Frontiers in Artificial Intelligence and Applications, vol. 185, pp. 131-153. IOS Press (2009) 1, 11

15. McMillan, K.L., Kuehlmann, A., Sagiv, M.: Generalizing DPLL to richer logics. In: Bouajjani, A., Maler, O. (eds.) Proceedings of the Twenty-First International Conference on Computer Aided Verification (CAV). Lecture Notes in Computer Science, vol. 5643, pp. 462-476. Springer (2009) 1

16. Nelson, G., Oppen, D.C.: Simplification by cooperating decision procedures. ACM Transactions on Programming Languages and Systems 1(2), 245-257 (1979) 2, 15, 16

17. Wang, C., Ivančić, F., Ganai, M., Gupta, A.: Deciding separation logic formulae by SAT and incremental negative cycle elimination. In: Sutcliffe, G., Voronkov, A. (eds.) Proceedings of the Twelfth International Conference on Logic for Programming, Artificial Intelligence, and Reasoning (LPAR). Lecture Notes in Artificial Intelligence, vol. 3835, pp. 322-336. Springer (2005) 1

18. Wolfman, S.A., Weld, D.S.: The LPSAT engine and its application to resource planning. In: Dean, T. (ed.) Proceedings of the Sixteenth International Joint Conference on Artificial Intelligence (IJCAI). vol. 1, pp. 310-316. Morgan Kaufmann Publishers (1999) 1

19. Zeljić, A., Wintersteiger, C.M., Rümmer, P.: Deciding bit-vector formulas with mcSAT. In: Creignou, N., Le Berre, D. (eds.) Proceedings of the Nineteenth International Conference on Theory and Applications of Satisfiability Testing (SAT). Lecture Notes in Computer Science, vol. 9710, pp. 249-266. Springer (2016) 2, 16 